\title{
Multi-mode Interferometer for Guided Matter Waves
}

\author{
Erika Andersson ${ }^{1}$, Tommaso Calarco ${ }^{2}$, Ron Folman ${ }^{3}$, Mauritz Andersson ${ }^{4}$, Björn Hessmo ${ }^{5}$, Jörg Schmiedmayer ${ }^{3}$ \\ 1 Department of Physics, Royal Institute of Technology, SE-10044 Stockholm, Sweden \\ (Present address: Department of Physics and Applied Physics, University of Strathclyde, Glasgow G4 0NG, Scotland) \\ 2 Institut für Theoretische Physik, Universität Innsbruck, A-6020 Innsbruck, Austria \\ European Centre for Theoretical Studies in Nuclear Physics and Related Areas, 38050 Villazzano (TN) Italy \\ 3 Physikalisches Institut, Universität Heidelberg, D-69120 Heidelberg Germany \\ ${ }^{4}$ Department of Quantum Chemistry, Uppsala University, S-75120 Uppsala, Sweden \\ ${ }^{5}$ Dept. of Microelectronics and Information Technology, Royal Institute of Technology, S-164 40 Kista, Sweden
}

(June 25, 2006)

\begin{abstract}
Atoms can be trapped and guided with electromagnetic fields, using nano-fabricated structures. We describe the fundamental features of an interferometer for guided matter waves, built of two combined Y-shaped beam splitters. We find that such a device is expected to exhibit high contrast fringes even in a multi-mode regime, analogous to a white light interferometer.
\end{abstract}

PACS number(s): 03.75.Be, 03.65.Nk

Interferometers are very sensitive devices, and have provided both insights into fundamental questions and valuable instruments for applications. The sensitivity of matter-wave interferometers [1] has been shown to be much better than that of light interferometers in several areas such as the observation of inertial effects [2]. Because of this high sensitivity, interferometers have to be built in a robust manner to be applicable. This could be achieved by guiding the matter waves with microfabricated structures, and by integration of the components into a single compact device, as has been done with optical devices.

In this Letter, we describe such an interferometer for matter waves propagating in a time-independent guiding potential, in analogy with propagation of light in optical fibers (for recent microtrap proposals see [3]). This interferometer has the surprising feature that interference is observed even if many levels in the guide are occupied, as is the case when the source is thermal, or for cold fermions, even below the Fermi temperature. The multimode interferometer is built by combining two Y-shaped beam splitters 胞, capable of coherently splitting or recombining many incoming transverse modes, and arranging the interferometer geometry so that all the different transverse states give the same phase shift pattern. The beam splitters are formed by splitting the atom guiding potential symmetrically into two identical output guides. This was recently demonstrated on an atom chip [4,5]; alternatively, this may be realized by optical confinement [6].

The analysis is done in two dimensions [7], similar to solid state electron interferometers [8]. The shape of the guiding potential in the transverse direction $x$ changes with the longitudinal coordinate $z$, from a single harmonic well of frequency $\omega$ to two wells of frequency $2 \omega$ separated by a distance $d(z)$, and then back again to a single guide (see Fig. 1a and b). Let us consider a particle entering in the transverse ground state and with longitudinal kinetic energy $E_{\text {kin }}$. In the limit where the

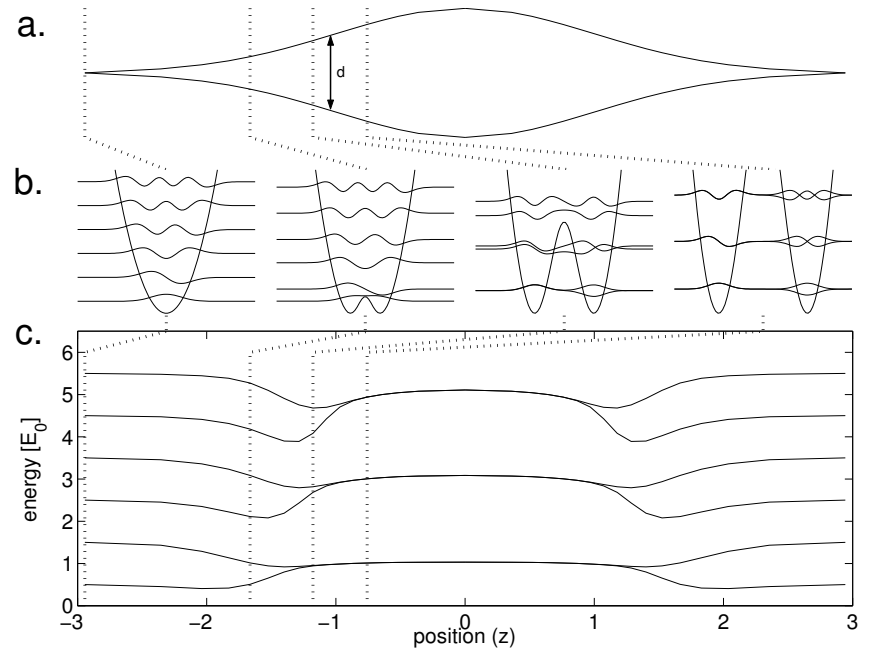

FIG. 1. The guided matter wave interferometer. a) Two beam splitters are joined together to form the interferometer. b) Transverse eigen-functions of the guiding potentials in various places along the first beam splitter. When the two outgoing guides are separated far enough, so that no tunneling between left and right occurs, the symmetric and the antisymmetric states become pairwise degenerate. c) Energy eigenvalues for the lowest transverse modes as they evolve along the interferometer.

atomic transverse motion in the guide (related to $\omega$ and the ground state size) is very fast with respect to the rate of transverse guide displacement $\frac{d}{d t} d(z)$ as seen by the moving atom (related to the beam splitter opening angle $\frac{d}{d z} d(z)$ and $\left.E_{\text {kin }}\right)$, the particle will adiabatically follow the lowest energy level throughout the interferometer. This corresponds to the condition $E_{\text {kin }}\left[\frac{d}{d z} d(z)\right]^{2} \ll \hbar \omega$. As it can be seen in Fig. 1b, when the two guide arms are far apart (i.e., when $d(z) \gg \sqrt{\hbar / m \omega}$ ), any eigenstate of the incoming guide potential evolves into a superposition of eigenstates $|n\rangle_{l}$ and $|n\rangle_{r}$, corresponding to the left and right arms, respectively: 


$$
\begin{gathered}
|2 n\rangle \longrightarrow \frac{1}{\sqrt{2}}\left[|n\rangle_{l}+|n\rangle_{r}\right] \\
|2 n+1\rangle \longrightarrow \frac{1}{\sqrt{2}}\left[|n\rangle_{l}-|n\rangle_{r}\right] .
\end{gathered}
$$

Levels $|2 n\rangle$ and $|2 n+1\rangle$ become practically degenerate inside the interferometer when the arms are widely split, as Fig. 1c shows. For example, it is easy to visualize how a transverse odd state $|1\rangle$ splits in the middle to become a superposition of two ground states having between them a $\pi$ phase, while the even state $|0\rangle$ does the same, but with a 0 relative phase. As the states become degenerate, an asymmetric perturbation (e.g., a differential phase shift) will couple the odd and even symmetries, thus inducing a mixing between the two states (see also [3]). Numerical two-dimensional wave-packet calculations for the lowest 35 modes [9] confirmed that no transitions between transverse states occur, as long as the motion is adiabatic in the sense discussed above, and as long as no asymmetric perturbation is present. In an ideal case, coherent splitting and recombination for all transverse modes can be achieved [10].

As mentioned, mixing within pairs of degenerate states inside the interferometer can occur if the wavefunction experiences a phase difference between the two arms. Let us therefore introduce a phase shift $\Delta \phi$ between the interferometer arms, either by making one arm longer by $\Delta l$, giving $\Delta \phi=k \Delta l$, where $\hbar k$ is the momentum in the longitudinal $(z)$ direction, or by applying an additional potential $U$, resulting in $\Delta \phi=\frac{m}{\hbar^{2} k} \int U d z$. Both phase shifts are independent of the transverse state in the guide, and they are dispersive, meaning that the resulting phase shift depends on $k$. In the following, we shall refer to the phase shift caused by a path length difference $\Delta l$.

Following equation 1 and its time inverse, an incoming even state is transformed as follows while transversing through the two beam splitters BS1 and BS2:

$$
\begin{aligned}
|2 n\rangle & \stackrel{B S 1+\Delta \phi}{\longrightarrow} \frac{1}{\sqrt{2}}\left[e^{i \Delta \phi / 2}|n\rangle_{l}+e^{-i \Delta \phi / 2}|n\rangle_{r}\right] \\
& \stackrel{B S 2}{\longrightarrow} \frac{1}{\sqrt{2}}[\cos (\Delta \phi / 2)|2 n\rangle+i \sin (\Delta \phi / 2)|2 n+1\rangle] .
\end{aligned}
$$

By taking into account the analogous transformation rule for the nearby odd state, we find that we can describe the interferometer in terms of a matrix

$$
\frac{1}{\sqrt{2}}\left(\begin{array}{ll}
\cos (\Delta \phi / 2) & i \sin (\Delta \phi / 2) \\
i \sin (\Delta \phi / 2) & \cos (\Delta \phi / 2)
\end{array}\right)
$$

in the basis of the two incoming and outgoing transverse eigenmodes $|2 n\rangle$ and $|2 n+1\rangle$. Figure 2 shows the above qualitative behavior in an actual simulation.

Let us now discuss the longitudinal degree of freedom. Consider a very cold wave packet with longitudinal kinetic energy $E_{k i n}=k^{2} \hbar^{2} / 2 m$, where $k$ is the mean longitudinal momentum of the wave packet. In the transverse direction it occupies the transverse ground state $|0\rangle$ of the incoming guide. We have chosen the ground state energy in the interferometer arms, $\hbar \omega^{\prime}$, to be twice that of the input and output guides, $\hbar \omega$ (see Fig. 1). This implies that if the longitudinal kinetic energy is too small, $E_{k i n}<\frac{1}{2} \hbar\left(\omega^{\prime}-\omega\right)=\frac{1}{2} \hbar \omega$, the wave packet will be reflected already at the first beam splitter. For $E_{k i n}>\frac{1}{2} \hbar \omega$, it will split between the two interferometer arms to occupy the transverse ground states in both arms, slowing down in the longitudinal direction to ensure energy conservation. If the wave packet experiences a phase shift $\Delta \phi=k \Delta l$ in one of the arms, part of it will, after the recombination at the second beam splitter, exit in the first excited transverse state $|1\rangle$ of the outgoing guide, as described by matrix (3), again provided the longitudinal kinetic energy is large enough. The phase shift is dependent on $k$, and thus the different $k$ components of the wave packet will obtain different phase shifts. The components with phase shifts close to $2 N \pi$ will exit in the transverse ground state, and those with phase shifts close to $(2 N+1) \pi$ will exit in the first excited state. If $\frac{1}{2} \hbar \omega<E_{k i n}<\hbar \omega$, the transition to the first excited transverse state will be forbidden, and this part of the wave packet will be reflected. If the kinetic energy of the wave packet fulfills $E_{k i n} \gg \hbar \omega$, transitions to the first excited transverse state at the recombination beam splitter are possible, and no sizeable back reflection occurs. The interference pattern may then be observed by looking at the populations in the states $|0\rangle$ and $|1\rangle$ in the outgoing guide, as presented in figure 2.

We note that the part of the wave packet making the transition to the state $|1\rangle$ will lose kinetic energy to compensate for the additional transverse energy $\hbar \omega$ needed for the transition. Therefore the part of the wave packet in state $|1\rangle$ will travel slower than the part in $|0\rangle$, by an amount $\Delta v \simeq \omega / k$. As explicitly shown in the third column of Fig. 目 for $\Delta \phi=3 \pi / 2$, the two outgoing components will separate longitudinally. Similarly, a wave packet entering in the first excited transverse state, acquiring an odd phase shift $(2 N+1) \pi$, will exit in the ground state, gaining potential energy, and propagating faster than the components exiting in the first excited transverse state. To observe this separation between the two outgoing parts of the wave packet, one has to introduce a pulsed source, which is what we will assume from now on.

The reasoning above is easily extended to higher transverse modes. Components of a wave packet acquiring an even phase shift will exit in the same transverse state as they entered, while components acquiring an odd phase shift will exit in the neighboring transverse state $(|2 n\rangle \rightarrow|2 n+1\rangle$, or $|2 n+1\rangle \rightarrow|2 n\rangle)$, lose (gain) kinetic energy accordingly, and propagate slower (faster) by the same $\Delta v$, independent of the transverse energy level. Thus, for a system of temperature $T$, filling $2 n+1$ transverse modes, the interferometer is actually composed of $n$ disjunct interferometers, all giving rise to the same longitudinal pattern.

If the energy spread of the longitudinal wave packet is large enough, $\Delta k \gg \pi / \Delta l$, a longitudinal interference pattern will form within the wave packet, as shown in 


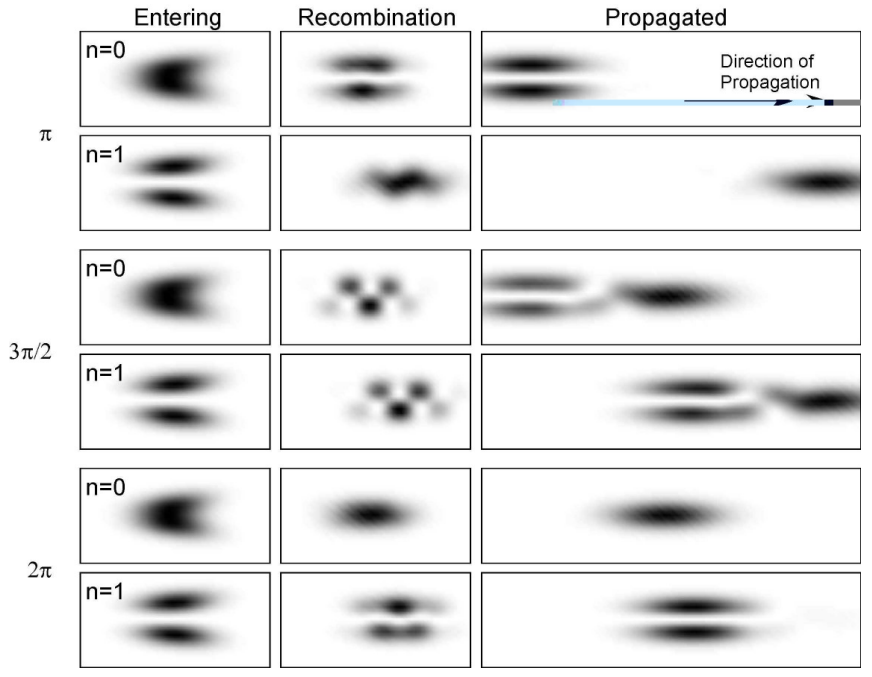

FIG. 2. Probability distributions of wave packets in the interferometer. The probability distributions are shown just before entering, right after exiting the interferometer, and after a rephasing time $t$. The incident wave packet is in the transverse ground state $(n=0)$ or first excited state $(n=1)$, the phase shift between the interferometer arms is $\pi, \frac{3 \pi}{2}$ or $2 \pi$. In this calculation, the wave packet is relatively narrow in momentum space, so that the whole wave packet obtains approximately the same $k$-dependent phase shift $\Delta \phi$. If $\Delta \phi$ is $2 \pi$, the wave packet exits in the same transverse state as it entered. If it is $\pi, n=0$ exits as $n=1$ and vice versa. For $\Delta \phi=3 \pi / 2$, the wave packet will exit in an equal superposition of the transverse ground state and first excited state. The separation of these two outgoing components after the rephasing time is due to energy conservation. In this example, the wave packets are sufficiently narrow in longitudinal momentum space, so that the whole wave packet acquires approximately the same $k$-dependent phase shift. The numerical calculation is done by solving the time-dependent Schrödinger equation in two dimensions using the split-operator method, for realistic guiding potentials.

Fig. 3. This pattern, shown here for the states $|0\rangle$ and $|1\rangle$, will be the same for all transverse states $|2 n\rangle$ and $|2 n+1\rangle$. The two density patterns will, at the exit of the interferometer, add up to the same wave packet shape one would expect if the interferometer was not there [11]. Looking at the total wave packet, not distinguishing between the different transverse levels, one only sees its envelope. The two transverse state components will, however, propagate with different velocities, as outlined above, and after some time they will rephase as shown in Fig. 3. This will happen once enough time has passed for $|\Delta v|$ to overcome the difference in the position of the pattern peaks. Since $|\Delta v|$ is independent of the incoming transverse mode, all patterns will re-phase at the same time and position, and a multi transverse mode operation could be achieved.

In an actual multi mode experiment, the simplest input state will be a thermal atomic cloud. Such a state is
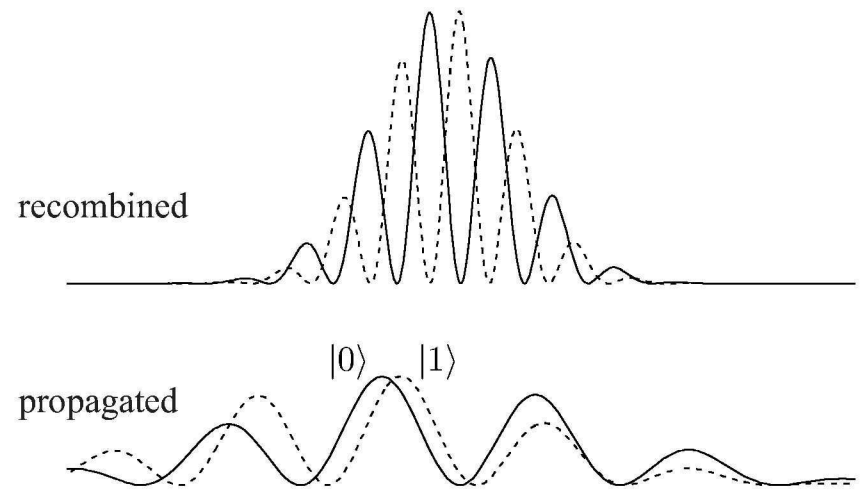

FIG. 3. The rephasing of the interference patterns created by a wave packet entering in the ground state $|0\rangle$. The full line shows the probability distribution of the transverse ground state $|0\rangle$, and the dashed line shows that of the first excited state $|1\rangle$. The wave packet width is chosen to have a phase difference greater than $2 \pi$ between its front end and tail. The top graph shows the wave packet just after recombination at the end of the interferometer, the lower graph after propagation, when the $|0\rangle$ part has caught up with the $|1\rangle$ part. The wave packet has also spread out.

described by a mixed density matrix. The longitudinal states are obtained in the following way: At the start of the experiment, we imagine a thermal atomic cloud trapped in both transverse and longitudinal directions, with transverse confinement by the same trapping frequency $\omega$ as in the incoming guide. In the longitudinal direction, the initial trap is approximated by an infinitely high box of width $L$. The states we consider are the eigenfunctions of this trap. At time $t=0$, one wall of the box is opened up and the longitudinal states start propagating in the positive $z$ direction according to their momentum distributions. Each longitudinal state can be approximated by a wave packet with mean momentum $k=(n+1) \pi / L$ and momentum spread $\Delta k=2 \sqrt{\pi} / L$ 114.

We carry out a numerical calculation of the interference pattern after the interferometer, starting from a thermal state as described. We take into account the $k$-dependent phase shifts acquired by the plane wave components of the longitudinal wave packets, resulting in transitions between different transverse states according to Eq. (3). The plane wave components are slowed down or sped up, if necessary, to ensure energy conservation. The emergence of an interference pattern, despite the incoherent sum over the transverse and longitudinal states, is confirmed by this numerical calculation. Figure 1 gives an example of an interference pattern obtained with a typical "hot" atom ensemble with a temperature of $200 \mu \mathrm{K}$ and guides with a trap frequency of $10^{5} \mathrm{~s}^{-1}$, already realized on an atom chip [12,13. Even though hundreds of transverse levels are populated, high contrast fringes are observed.

To summarize, we have shown that a multi-mode in- 


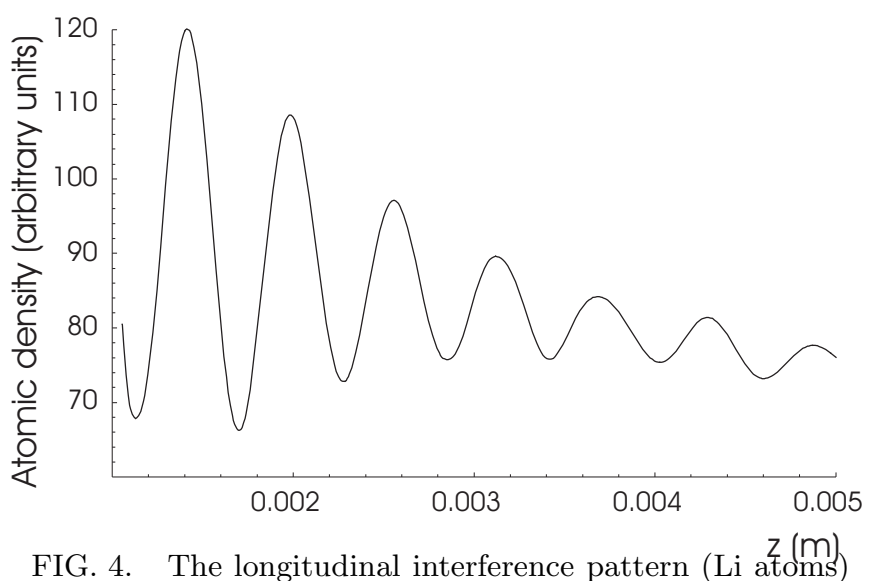

FIG. 4. The longitudinal interference pattern ( $\mathrm{Li}$ atoms) as a function of $z$. The interferometer is $1 \mathrm{~mm}$ long with the source at its beginning. The transverse trapping frequency is $\omega=0.1 \times 10^{6} \mathrm{~s}^{-1}$ and the length of the source trap is $L=100 \mu \mathrm{m}$. The path difference between the arms is $\Delta l=2 \mu \mathrm{m}$. The time elapsed since the release of the atoms from the trap is $20 \mathrm{~ms}$. The periodicity of the interference pattern is about $550 \mu \mathrm{m}$, consistent with $\cos ^{2}[m \Delta l z /(2 \hbar t)]$, the analytically calculated modulation for a single mode in the limit of an initially narrow width source wave packet and a sufficiently long propagation time. The intensity is linearly dependent on the intensity of the source.

terferometer may be realized in a two-dimensional geometry, using two symmetric Y-shaped beam splitters. Furthermore, though beyond the scope of the present work, we expect three-dimensional evolution to exhibit qualitatively the same behavior under certain conditions. As Y-shaped microfabricated beam splitters have been realized, we expect the road to be open for the experimental realization of robust guided matter wave interferometers.

We would like to thank Peter Zoller for enlightening discussions. R.F. is grateful to Yoseph Imry for his insight into mesoscopic systems. E.A. would like to thank Helmut Ritsch for his kind hospitality. This work was supported by the Austrian Science Foundation (FWF), project SFB 15-07, by the Istituto Trentino di Cultura (ITC) and by the European Union, contract Nr. IST-1999-11055 (ACQUIRE), HPMF-CT1999-00211 and HPMF-CT-1999-00235.

[1] Proceedings of the International Workshop on Matter Wave Interferometry, ed. by G. Badurek, H. Rauch, and A. Zeilinger [Physica (Amsterdam) 151B, No. 1-2 (1988)]; Atom Interferometry, ed. by P. Berman, Academic Press (1997).

[2] For example see A. Peters, K.Y. Chung and S. Chu, Nature 400, 849 (1999); T.L. Gustavson, A. Landragin and M.A. Kasevich, J. Class. and Quant. Grav. 17, 2385 (2000); and references there in.

[3] Recently, several proposals for matter-wave interferometers have been made in the context of splitting and combining a microtrap ground state with a time dependent potential: E.A. Hinds, C.J. Vale and M.B. Boshier, Phys.
Rev. Lett. 86, 1462 (2001); W. Hänsel, J. Reichel, P. Hommelhoff and T.W.Hänsch, quant-ph/0106162.

[4] D. Cassettari, B. Hessmo, R. Folman, T. Maier, J. Schmiedmayer, Phys. Rev. Lett. 85, 5483 (2000).

[5] D. Müller, et al., Phys. Rev. A 63, 041602(R) (2001).

[6] O. Houde et al., Phys. Rev. Lett. 85, 5543 (2000).

[7] In 2D confinement the out-of-plane transverse dimension is either subject to a much stronger confinement, or the potential is separable. For an experimental realization, see H. Gauck et al. Phys. Rev. Lett. 81, 5298 (1998); T. Pfau priv. comm. (2000); E.A. Hinds, M.G. Boshier, I.G. Hughes Phys. Rev. Lett. 80, 645 (1998); R.J.C. Spreeuw, et al. Phys. Rev. A 61, 053604 (2000).

[8] E. Buks et al., Nature 391, 871-874 (1998).

[9] The numerical calculation is based on the split-operator method with a pseudospectral method for derivatives. See e.g. M. Feit, J. Fleck Jr, and A. Steiger, J. Comput. Phys. 47, 412 (1982); B. M. Garraway, K.-A. Suominen, Rep. Prog. Phys 58, 365 (1995).

[10] This is an advantage over four-port beam splitter designs relying on tunneling through a barrier between two guides - see E. Andersson, M. T. Fontenelle, and S. Stenholm, Phys. Rev. A 59, 3841 (1999). In the latter, the splitting ratios for incoming wave packets are very different for different transverse modes, since the tunneling probability depends strongly on the energy of the particle. Even for a single mode, the splitting amplitudes, determined by the barrier width and height, are extremely sensitive to experimental noise. On the contrary, the operation of the Y-shaped beam splitter, is based on a symmetric barrier, rising in the center of the transverse wave function, splitting it independently of the mode number.

[11] This is only approximately true, because inside the interferometer there are different propagation velocities due to the different potential energies of even and odd incoming states. In the calculations, this small effect is included.

[12] R. Folman et al., Phys. Rev. Lett. 84, 4749 (2000); current traps on our chips achieve $\omega>10^{6} \mathrm{~s}^{-1}$.

[13] For other atom chip experiments see J. Reichel et al., Phys. Rev. Lett. 83, 3398 (1999); D. Müller et al., Phys. Rev. Lett. 83, 5194 (1999); N.H. Dekker, et al., Phys. Rev. Lett. 83, 3398 (1999).

[14] In this model, and for a reasonable source size $L$, the wave packet energy spread will be relatively small and no complete pattern of several oscillations is expected to be observable within a single packet. This however, does not alter the final result as no coherence between different longitudinal $k$-components is needed. The only coherence evoked in the interferometer is that between the right and left paths, which in turn requires that a single wave packet have a coherence length longer than the path length difference $\Delta l$. The number of observed oscillations will then only depend on the longitudinal energy spread of the source. 